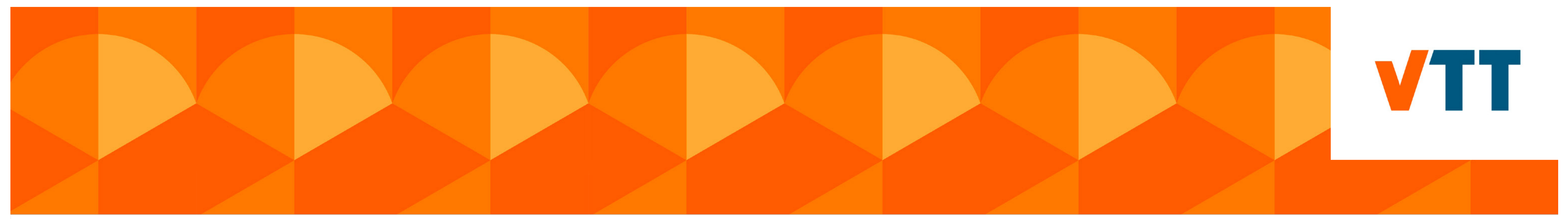

\title{
Making the invisible visible
}

\section{Towards strategic measures of research and technology organisations (RTOs)}

\author{
Kirsi Hyytinen, Katri Kallio, Olli Kuusisto \& Arho Suominen
}

VTT Technical Research Centre of Finland Ltd

\section{RTOs are tackling with systemic and complex challenges}

There are no simple solutions to complex societal problems. Modern innovation theories emphasizes that tackling system level problems requires considering three perspectives:

1) Solutions require deep integration of technologies and service-based novelties.

2) Collaboration between multiple actors from different sectors of society is required.

3) Developing and disseminating solutions requires understanding of customer and citizen needs and the policy-making context.

\section{Current innovation measures do not make the impact visible}

Target of RTOs is to solve broad societal challenges. However, current innovation indicators do not capture RTOs' multiple roles and impact in systemic environment.

There are two main reasons why traditional evaluation and measures fail. The focus is on:

- techno-economic aspects of innovation and impact, which are not able to capture the reality of innovations (e.g. system, social and service innovations) and "hidden performance".

- input and direct outputs. Therefore we lack data about impact.

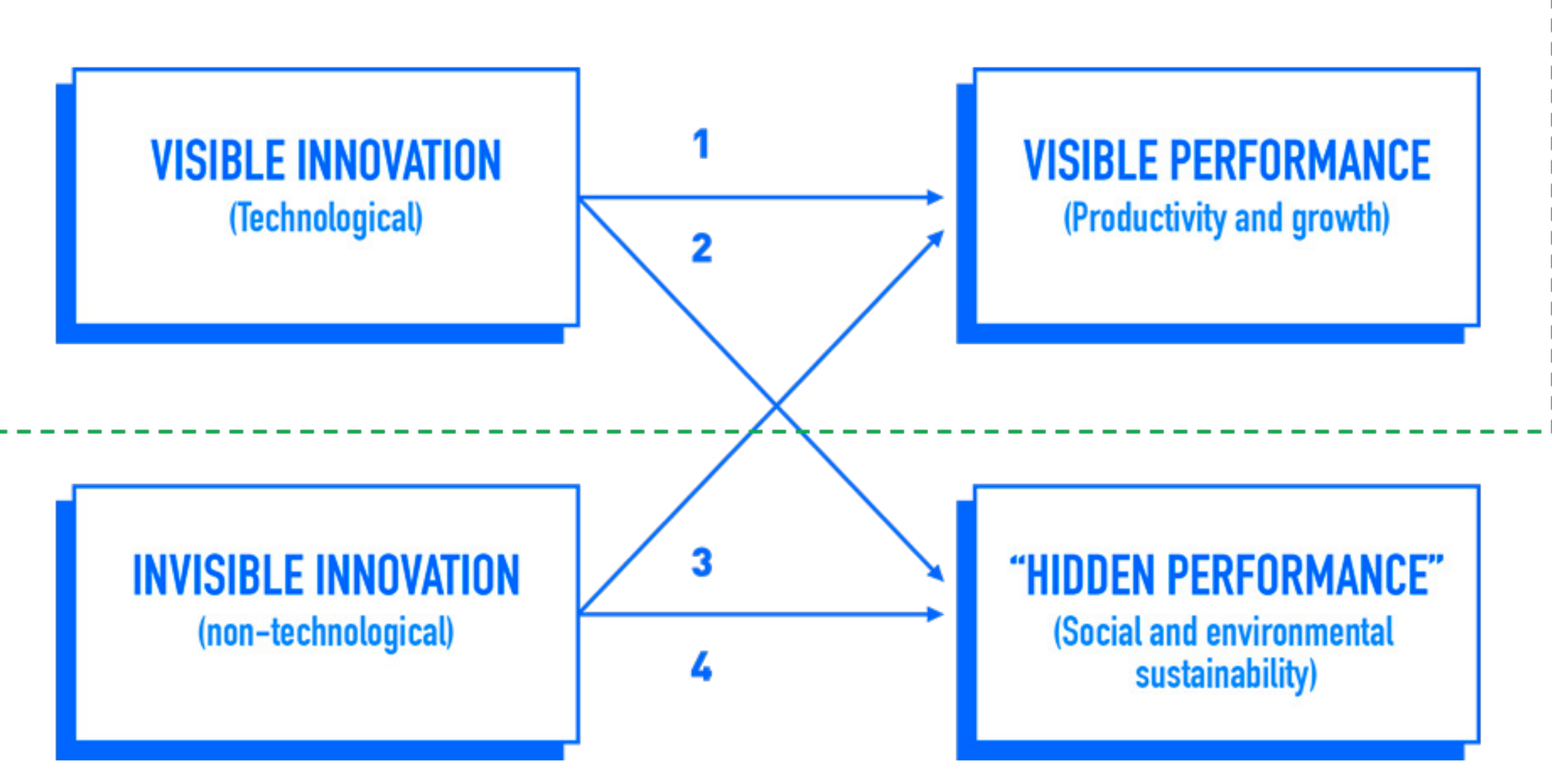

Figure 1. The focus in the impact analysis is typically in the linkages between the visible innovation and visible performance, which do not capture the nontechnological innovation and hidden performance of innovations. (Djellal \& Gallouj, 2010).

Biased information may cause inaccurate analysis and interpretations and lead to inappropriate decisions. In order to paint a truthful and comprehensive picture of RTOs' impact, and to provide better information for managing and learning, we need diversified evaluation approach and measures.

\section{VTT's strategic evaluation framework}

VTT's new strategic evaluation framework and related measures of success (KPIs) integrate multi-criteria approach of innovation to the traditional balanced scorecard (BSC). The framework expands traditional innovation indicators towards broader societal transformations in accordance with VTT's strategy. They make VTT's long-term impact visible in four categories:

\section{1) Benefit for society}

2) Benefit for customers

3) Excellence in everything we do

4) Sufficiency of financial resources

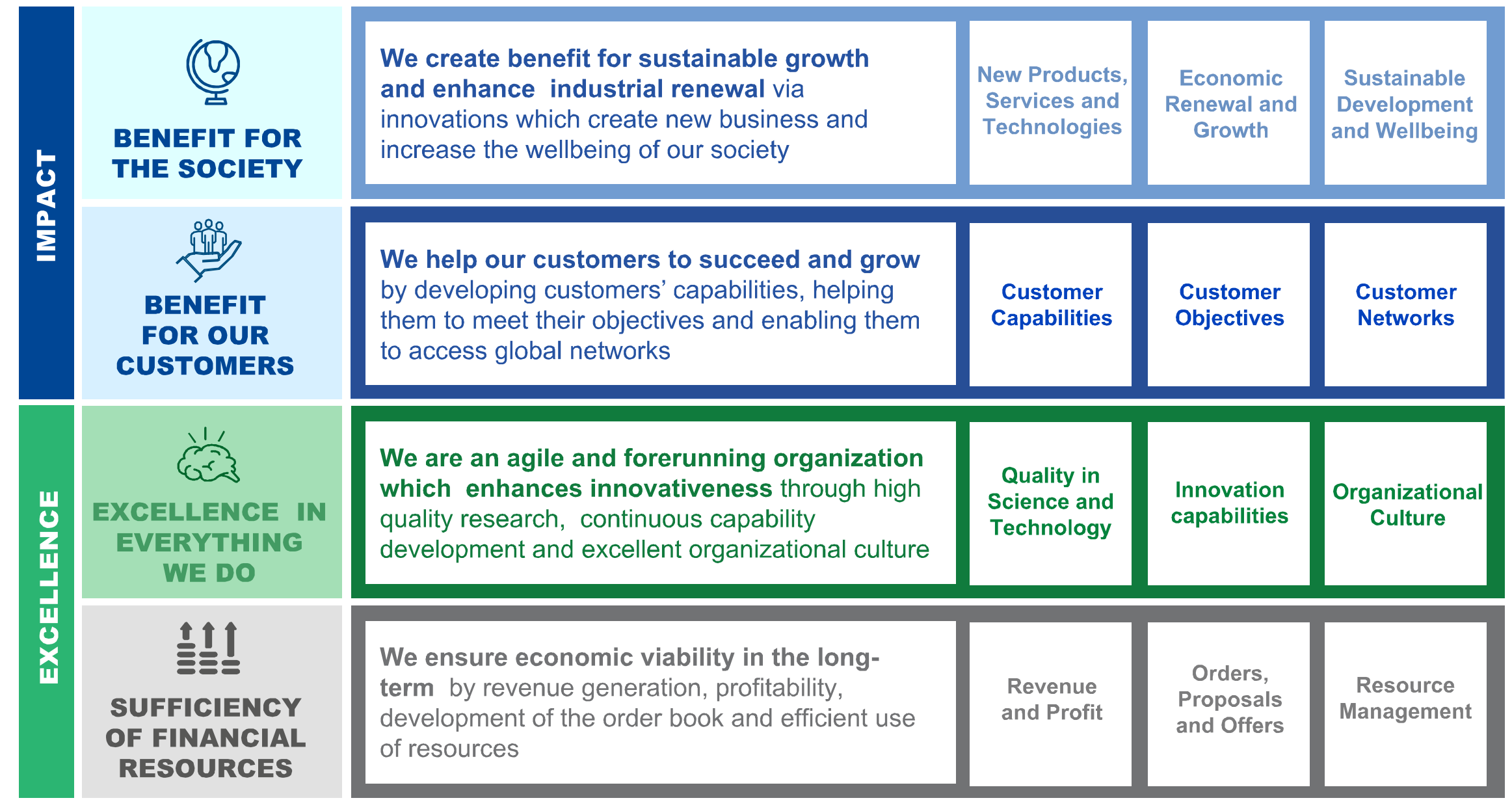

Figure 2. Framework to measure success at VTT.

The long-term societal impact is based on VTT's ability to create customer impact in shorter term. To ensure this, we need to support customers' success and growth and actively create new partner networks. A prerequisite for creating impact is high-level competence in research and innovation. Finally, operative excellence and financial profitability should secure the balance of VTT's finance in the long run.

Conclusions

- To make the invisible visible we need more diversified evaluation approach and indicators which enable to analyse RTOs impact in the systemic and complex environment.

- RTOs' impact cannot be demonstrated with one - or even a few single indicators: a comprehensive picture of impact requires multi-criteria approach, a variety of indicators and data gathering from multiple sources.

- Policy measures and evaluation practices could be updated to perceive the significance and 'hidden performance' of innovations in systemic context: innovations do not emerge without evaluation mechanisms that support their creation.

\section{VTT - beyond the obvious}

www.vttresearch.com

\section{Contact}

Kirsi Hyytinen

Tel. +358405818495

Kirsi.Hyytinen@vtt.fi 